\title{
Deletion of Pten in the mouse enteric nervous system induces ganglioneuromatosis and mimics intestinal pseudoobstruction
}

\author{
Isabel Puig,,1,2 Delphine Champeval,1,2 Pascal De Santa Barbara, ${ }^{3}$ \\ Francis Jaubert, ${ }^{4,5}$ Stanislas Lyonnet, ${ }^{4,5}$ and Lionel Larue ${ }^{1,2}$ \\ ${ }^{1}$ Institut Curie, Centre de Recherche, Developmental Genetics of Melanocytes, Orsay, France. \\ ${ }^{2}$ CNRS UMR146, Orsay, France. ${ }^{3}$ INSERM ERI 25, Muscle and Pathologies, Montpellier Cedex 05, France. \\ ${ }^{4}$ INSERM U-781, Hôpital Necker-Enfants Malades, Paris, France. ${ }^{5}$ Université Paris-Descartes, Paris, France.
}

\begin{abstract}
Intestinal ganglioneuromatosis is a benign proliferation of nerve ganglion cells, nerve fibers, and supporting cells of the enteric nervous system (ENS) that can result in abnormally large enteric neuronal cells (ENCs) in the myenteric plexus and chronic intestinal pseudoobstruction (CIPO). As phosphatase and tensin homolog deleted on chromosome 10 (PTEN) is a phosphatase that is critical for controlling cell growth, proliferation, and death, we investigated the role of PTEN in the ENS by generating mice with an embryonic, ENC-selective deletion within the Pten locus. Mutant mice died 2 to 3 weeks after birth, with clinical signs of CIPO and hyperplasia and hypertrophy of ENCs resulting from increased activity of the PI3K/PTEN-AKT-S6K signaling pathway. Further analysis revealed that PTEN was only expressed in developing mouse embryonic ENCs from E15.5 and that the rate of ENC proliferation decreased once PTEN was expressed. Specific deletion of the Pten gene in ENCs therefore induced hyperplasia and hypertrophy in the later stages of embryogenesis. This phenotype was reversed by administration of a pharmacological inhibitor of AKT. In some human ganglioneuromatosis forms of CIPO, PTEN expression was found to be abnormally low and S6 phosphorylation increased. Our study thus reveals that loss of PTEN disrupts development of the ENS and identifies the PI3K/PTEN-AKT-S6K signaling pathway as a potential therapeutic target for ganglioneuromatosis forms of CIPO.
\end{abstract}

\section{Introduction}

The enteric nervous system (ENS) regulates peristalsis, secretions, blood supply, and immune responses in the intestinal tract (1). The mammalian ENS is composed of a large number of neurons and glia that are organized into enteric ganglia distributed throughout the gut wall (2). ENS cells cluster into 2 plexi: the myenteric plexus develops first and is situated between the inner circular and outer longitudinal layers of the muscularis propia; the submucosal plexus forms later during gestation and is positioned between the muscularis propia and the muscularis mucosa (3).

Neural crest cells develop from the dorsal part of the neural tube of embryos. They migrate into most of the peripheral regions to produce various derivatives including skin melanocytes and the ENS. In the vagal region, the ENS progenitors, enteric neural crest-derived cells (ENCCs), and their derivatives proliferate actively to expand the relatively small pool of progenitors that invade the foregut; they thereby generate the millions of enteric neurons and glia that are present in the adult intestine (4). A fully colonized gut, with the appropriate number of neuronal and glial cells, is required for integrated peristaltic activity of the gut wall.

Many pediatric consultations are due to ENS disorders resulting from a number of neurocristopathies (5). Chronic intestinal pseudoobstruction (CIPO) is a rare, severe, and disabling disorder characterized by repetitive episodes or continuous symptoms of bowel obstruction; it is associated with substantial morbidity and mortality. There are multiple forms and classifications of CIPO,

Conflict of interest: The authors have declared that no conflict of interest exists. Citation for this article: J. Clin. Invest. 119:3586-3596 (2009). doi:10.1172/JCI39929. but all are associated with neuronal or muscular defects. Disorders of neuronal density (aganglionosis, hypoganglionosis, or hyperganglionosis) have been associated with a neuronal form of CIPO (6).

The most common and best understood type of enteric neuropathy commonly associated with CIPO is Hirschsprung disease (HSCR). HSCR is a congenital disorder characterized by the absence of ganglion cells in the distal rectum and a variable length of contiguous intestine, leading to tonic contraction of the affected segment, intestinal pseudoobstruction, and massive distension of the proximal bowel, resulting in megacolon (7). There are a number of experimental models of HSCR, including loss-offunction mutants of RET protooncogene (RET), its coreceptors GFR $\alpha 1$ and GFR $\alpha 2$, and its ligand GDNF as well as endothelin receptor $\mathrm{B}(\mathrm{EDNRB})$ and its ligand $\mathrm{EDN} 3$, SRY (sex-determining region) box 10 (SOX10), and other less well-studied proteins, such as IHH and ITGB1 (reviewed in ref. 8). Reduced numbers of enteric progenitors and disruption of their differentiation and/or migration in these mutant mouse models lead to an abnormally small number of neurons along the whole length of the gut or to aganglionosis of the terminal gut; the consequence is, in some cases, an intestinal pseudoobstruction.

CIPO may also be associated with hyperganglionosis. At least 2 disorders are associated with clinical features of CIPO: intestinal neuronal dysplasia B (IND B) and ganglioneuromatosis (GNM). One consistent characteristic of human IND B is hyperplasia of submucosal and mucosal portions of the $\operatorname{ENS}(9,10)$. However, considerable debate surrounds the existence and/or importance of IND B (11). The motor activity of the intestinal tract is controlled in a complex manner by the balance between the inhibitory and excitatory neurons' activities. The hyperinnervation in IND B cases 


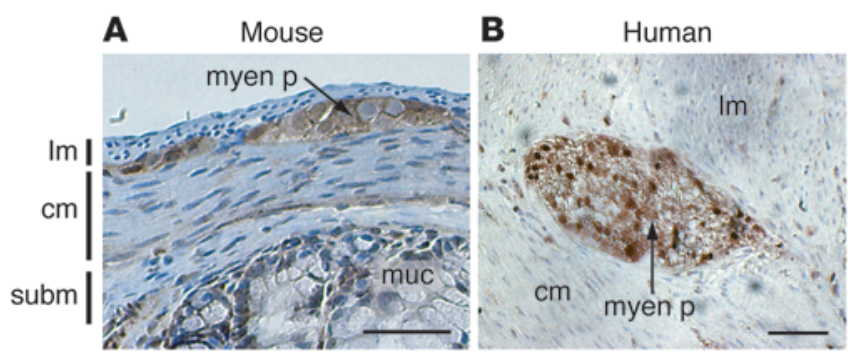

Figure 1

PTEN is present in the adult mouse and human ENS. Transverse sections of normal mouse $(\mathbf{A})$ and human $(\mathbf{B})$ colon were immunostained with antibody directed against PTEN. myen $\mathrm{p}$, myenteric plexus; Im, longitudinal muscle; cm, circular muscle; subm, submucosa; muc, mucosa. Scale bars: $50 \mu \mathrm{m}$.

results in both inappropriate activation of enteric neurons and a functional abnormality of the colon (12). Two IND mouse models, HOX11L1 $1^{-/-}$(where HOX11L1 indicates T cell leukemia homeobox 2) (13) and SPRY-2-/- (where SPRY-2 indicates SPROUTY-2) (14), demonstrate hyperplasia of the myenteric plexus leading to a megacolon or intestinal pseudoobstruction. GNM is a benign proliferation of nerve ganglion cells, nerve fibers, and supporting cells of the ENS (15). In humans, common symptoms of GNM include constipation, diarrhea, and bleeding. GNM is also classically associated with multiple endocrine neoplasia type 2B (MEN2B) and with neurofibromatosis 1 (NF1), characterized by germ-line RET and $N F 1$ gene mutations, respectively $(16,17)$. It has been ascribed to cellular hypertrophy and hyperplasia (18). Unfortunately, there is no mouse model for GNM.

Phosphatase and tensin homolog deleted on chromosome 10 (PTEN) regulates cell size and cell proliferation (19). It is a dualspecificity phosphatase that is able to dephosphorylate both protein (20) and phospholipid substrates (21). PTEN dephosphorylates phosphatidylinositol-3,4,5-triphosphate (PIP3), a product of PI3K (21-23), and thereby inhibits the growth factor signals transduced through PI3K. Consequently, it has a wide range of effects on cell growth and proliferation, processes involved in normal development. PTEN deficiency leads to accumulation of PIP3, which in turn activates several signaling molecules including AKT and S6K (19). Moreover, PTEN deficiency can alter the MAPK/ERK signaling pathway (24). Consequently, crosstalk between the PTEN and Ret/MAPK signaling pathways has been demonstrated (25).

The PTEN tumor suppressor gene is one of the genes most frequently mutated/deleted in human cancer (26). Furthermore, deletion of PTEN in mice reveals that it is essential for development: PTEN-null embryos die early during embryogenesis (22, 27-29). Much of our current knowledge about the functions of PTEN during development has therefore been acquired from animals with tissue-specific PTEN deletions generated with the Cre-loxP system. The deletion of PTEN specifically in the brain during midembryonic development leads to a doubling of brain size and weight, similar to the macrocephaly observed in humans with inherited PTEN deletions/mutations (30); the enlarged brain results from enlarged cell size, increased cell proliferation, and decreased cell death (31).

PTEN is produced in embryonic stem cells (23). In situ hybridization and immunohistochemical analyses in the mouse indicate that PTEN is expressed in both extraembryonic and embryonic tissues $(28,32)$. Within the embryo itself, PTEN is expressed ubiquitously during the early stage of embryonic development (E7.0-E11.0), but its distribution becomes more restricted to particular tissues and organs during fetal development (E15.0-E19.0) (28). Immunohistochemical analyses in humans show that PTEN is abundant in the nervous plexus of the gastrointestinal system during fetal development (33).

As PTEN is central to the control of cell growth, proliferation, and death and to the regulation of normal development and physiology, it seemed likely that PTEN plays a role during ENS development. We therefore deleted the PTEN gene in ENCCs. Tyr:Cre transgenic mice were able to recombine genes in ENCCs, giving rise to enteric neuronal cells (ENCs) of the ENS (refs. 34, 35, and Supplemental Figure 1; supplemental material available online with this article; doi:10.1172/JCI39929DS1).

Here, we show that the loss of PTEN disrupts the correct development of the ENS and that the PI3K/PTEN-AKT-S6K signaling pathway is clearly involved in the pathogenesis of GNM; thus, PI3K/PTEN-AKT-S6K signaling is a candidate therapeutic target in human CIPO.

\section{Results}

PTEN is expressed in the adult mouse and human ENS. We first used immunohistochemistry to analyze the distribution of PTEN in colon sections of adult mouse and human (Figure 1). PTEN was abundant in the myenteric plexus of the mouse and human ENS; in contrast, there was no PTEN staining in smooth muscle cells of the longitudinal and circular muscle layers located around the myenteric plexus. The distribution of PTEN in the ENS appeared to be conserved between these 2 different species.

Tyr: Cre ${ }^{\circ} ; P T E N^{F / F}$ mice die of an intestinal pseudoobstruction. Conditional PTEN-knockout mice were generated previously by flanking exon 5, encoding the phosphatase domain of PTEN, with loxP sequences (30). Heterozygous Tyr:Cre ${ }^{\circ} ; \mathrm{PTEN}^{F /+}$ mice (referred to herein as HT mice) obtained after appropriate crosses did not present any obvious phenotype. These mice were crossed with ${ }^{\circ}{ }^{\circ} ; P T E N^{F / F}$ mice to produce homozygous Tyr:Cre $/{ }^{\circ} ; P T E N^{F / F}$ mice (referred to herein as HM or PTEN mice). These crosses led to a normal Mendelian segregation (Supplemental Table 1). Tyrosinase is active in melanoblasts (34), in a subset of smooth muscle precursors forming the ductus arteriosum, in the heart, and in ENS cells (35). As expected, hyperpigmentation was observed in the tail, pinna, paws, skin, and olfactory bulb in HM mice (Supplemental Figure 2). We did not observe any obvious differences between HM and WT hearts and brains (Supplemental Figure 3). HM mice died younger than their WT and HT littermates (Figure 2A): HM mice died between 13 and 20 days after birth (P13-P20), with a 100\% penetrance. On P2, HM mice were the same size and weight as WT mice (Figure 2B). However, over the following 20 days, although they grew, the weight of HM mice lagged significantly behind that of WT/HT mice. One or 2 days before their deaths, HM mice were prostrated due to their abdominal pain; they did not eat and started losing weight such that, by the time of death, HM mice were smaller than WT littermates (Figure 2B). HM mice and their WT siblings were sacrificed and autopsied at the same time (Figure 2, $\mathrm{C}-\mathrm{F}$ ). HM mice exhibited a dilatation of the cecum, some intraluminal bubbles all along the gastrointestinal tract, and an empty colon. The gastrointestinal tract was opened transversally to check the absence of mechanical obstruction. We observed intestinal perforation at the ileum in some cases. All these clinical signs were suggestive of intestinal pseudoobstruction. 
A
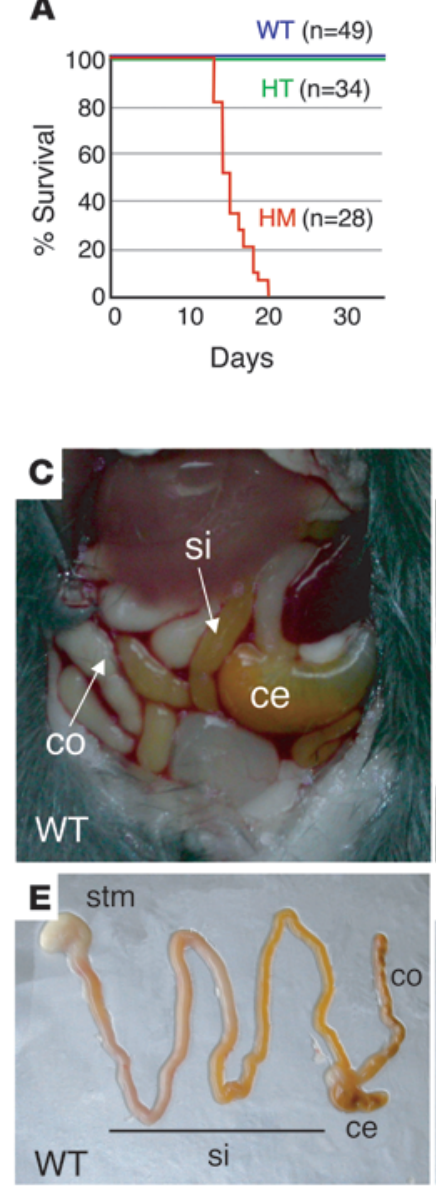

B
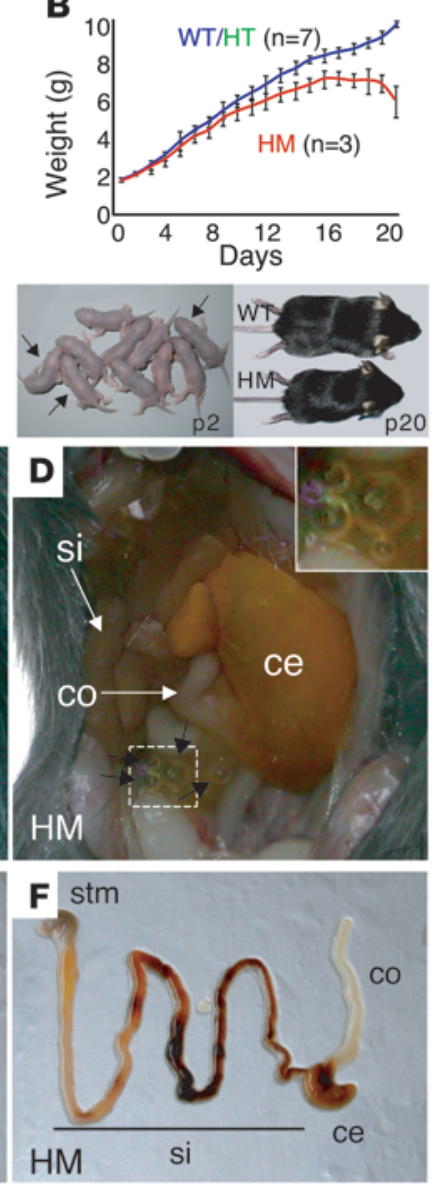

Loss of PTEN in ENCs leads to hypertrophy and hyperplasia of the ENS. Relative to WT mice, all HM mice suffered hypertrophy and hyperplasia of the myenteric and submucosal plexus in the ENS by 2 weeks of age (Figure 3A and Supplemental Figure 4). Analysis of standard neuronal markers, including neuron-specific class III $\beta$-tubulin (TUJ1) and neurofilaments (NF), and the neuronal activity marker synaptophysin (Syn) demonstrated unambiguous hyperplasia of ENCs (TUJ1 and NF) and higher neuronal activity (Syn) in HM than in WT mice (Figure 3A). To confirm these results, we isolated the tunica muscularis, which includes the ENS, by cleanly dissecting away the mucosa layer and tested protein lysates for TUJ1 by immunoblotting (Supplemental Figure 5). TUJ1 protein was more abundant in the tunica muscularis from 2-week-old HM mice than in control mice. As expected, we obtained similar results in analyses of glial cells, which form the ENS with ENCs, using immunohistochemistry, immunofluorescence, and immunoblot for the glial marker glial fibrillary acidic protein (GFAP) (Supplemental Figure 6). We measured the sizes of neuronal cells in colon sections from WT, HT, and HM mice. Cells in HM mice were double the size of their WT and HT littermates (Figure 3B). We counted the number of ENCs of the myenteric plexus along the entire gastrointestinal tract in sections of the proximal, middle, and distal small intestine and colon of WT, HT, and HM mice stained for TUJ1 expression and counterstained with DAPI (Figure 3C). In WT mice, there were more ENCs of the myenteric plexus in the colon than in the small intestine. Similar results have been previously reported using cuprolinic blue staining (36). In contrast, cell counts were 3 times higher in HM

\section{Figure 2}

HM mice die between 2 and 3 weeks of age with an intestinal pseudoobstruction. (A) Kaplan-Meier graphs comparing the survival of $\mathrm{HM}$ mice with that of $\mathrm{HT}$ and WT littermate controls $\left(\% ; P T E N N^{F /+}\right.$ and $\% ; P T E N F / F)$. The number of animals followed is indicated beside the curves. (B) Weight curves for HM mice and WT/HT littermate controls show a clear difference from p12. HT and WT mice had similar weights throughout their lives. Three HM pups (bottom left at p2) derived from $\% ; P T E N F / F \times H T$ genitors are shown (black arrow). By the time that mice were killed (bottom right at p20), the size differences between HM mice and WT littermates were easily observed. Error bars represent SEM. (C and D) Macroscopic views of WT (C) and HM (D) guts at p15. All HM mice displayed bubbles in the small intestine (black arrows), and some exhibited dilatation of the cecum. Original magnification, $\times 2.5$ (D, inset). Such abnormalities were not observed in WT or HT mice. ( $E$ and $\mathbf{F}$ ) The entire gastrointestinal tract dissected from WT (E) and HM (F) mice at p15. The colon of the HM mouse was empty. stm, stomach; si, small intestine; ce, cecum; co, large intestine.

mice than in WT and HT mice. The number of ENCs did not differ significantly between WT and HT mice throughout the small intestine and colon. The difference in number of ENCs between HM and WT mice was the same for all parts of the small intestine and colon. This suggests a homogeneous hyperplasia of the ENS throughout the gastrointestinal tract (Figure 3C).

To confirm that the phenotype was cell autonomous, we checked to make sure that PTEN exon 5 was deleted in the ENS of HM mice. We isolated the tunica muscularis from the colon and extracted the DNA. We then used PCR to amplify the fragment corresponding to floxed and defloxed PTEN gene and showed that PTEN was defloxed in HM tunica muscularis cells (Figure 4A). Moreover, we confirmed that enteric neuronal and glial cells from HM mice contained no detectable PTEN protein (Figure 4B and Supplemental Figure 6A).

Hypertrophy and hyperplasia of the ENS in adult HM mice is regulated by the AKT-mTOR signaling pathway. We studied the molecular mechanisms that lead to hyperplasia and hypertrophy in the ENS of HM mice. First, we examined the phosphorylation status of known downstream effectors of the PTEN signaling pathway, including S6 (mTOR signaling pathway), which is phosphorylated by S6K (also known as Rps6kb1), AKT (PI3K signaling pathway), and ERK (MAPK signaling pathway) (37), which have all been implicated in the regulation of cell growth and cell proliferation. Transverse sections of $\mathrm{p} 15$ colon from WT and HM mice were immunostained for p-S6, p-AKT1, and p-ERK1/2 (Figure 5A). Protein lysates from WT and HM tunica muscularis of $\mathrm{p} 15$ colon were also subjected to immunoblotting (Figure 5B). S6 and AKT phosphorylation levels were clearly higher in HM than in WT samples, this effect being particularly marked for AKT. However, ERK1/2 phosphorylation was not significantly different in WT and HM samples. These findings suggest that the AKT/mTOR signaling pathway, but not the MAPK signaling pathway, regulates the hypertrophy and hyperplasia associated with PTEN deletion in ENCs of the ENS. It has been previously shown that the lipid phosphatase activity of PTEN leads to cell-cycle arrest through upregulation of $\mathrm{p} 27$, the negative regulator of $\mathrm{CDK}$ (38). We therefore immunostained transverse colon sections for p27. p27 protein levels in the ENS of HM mice were slightly lower than those in WT mice (Supplemental Figure 7).

PTEN loss from ENCs results in continued proliferation of ENCs at late stages of development. Tyr:Cre;PTEN $N^{F / F}$ mice have a marked phenotype, suggestive of abnormal development of the ENS during embryogenesis. We investigated the temporal pattern of PTEN 
A

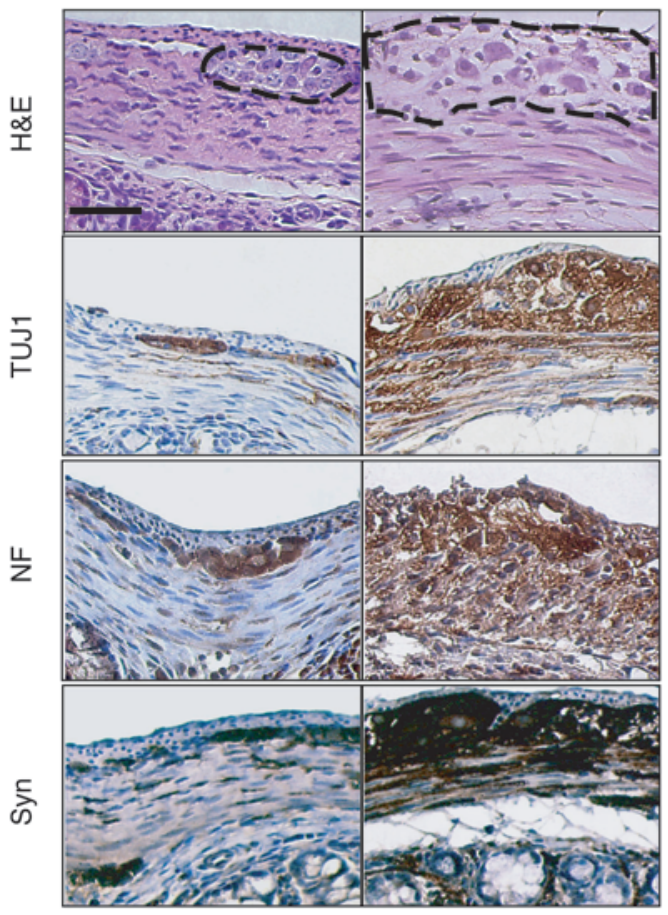

B

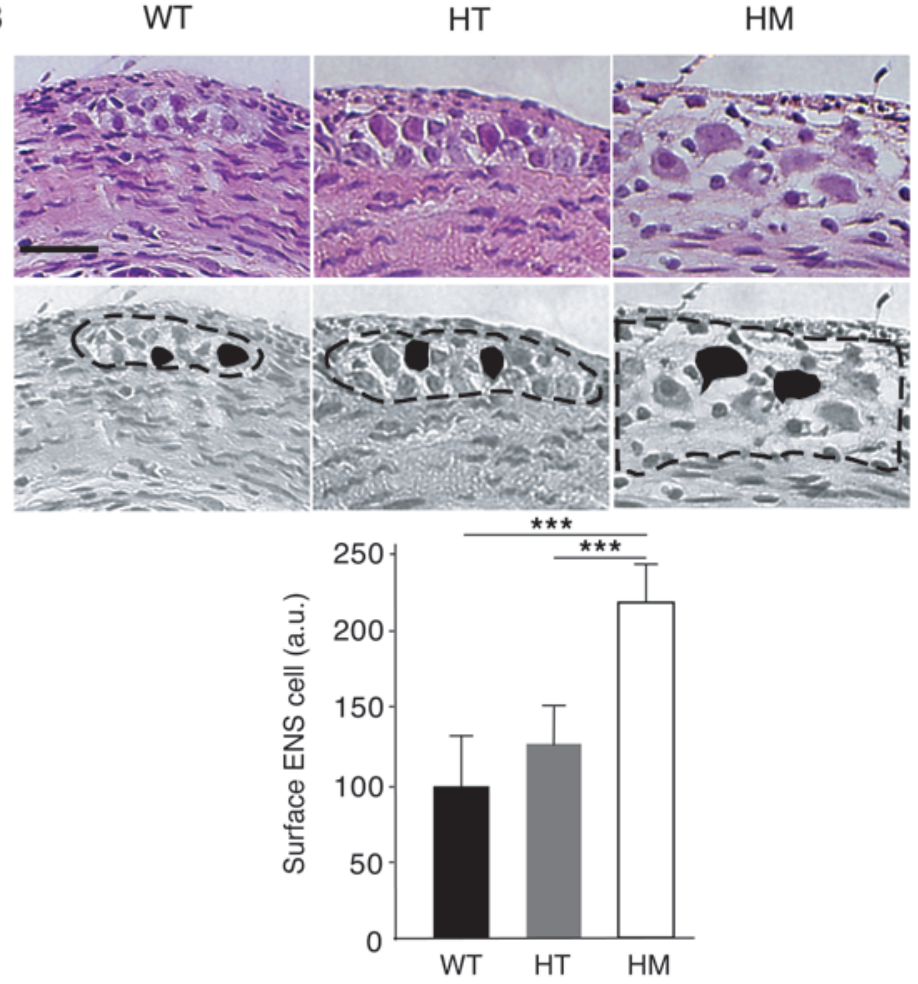

C WT

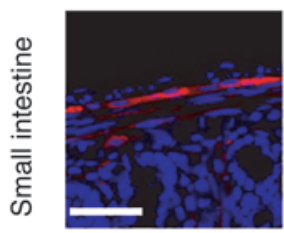

응
HT
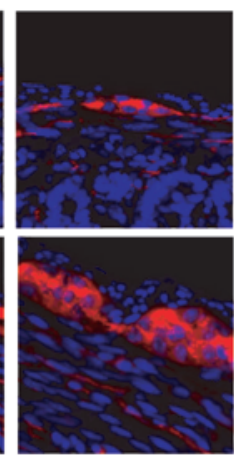

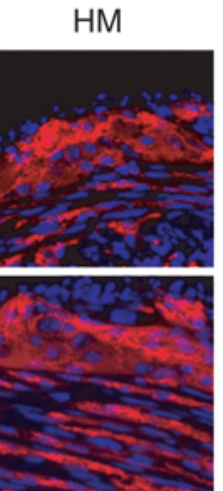

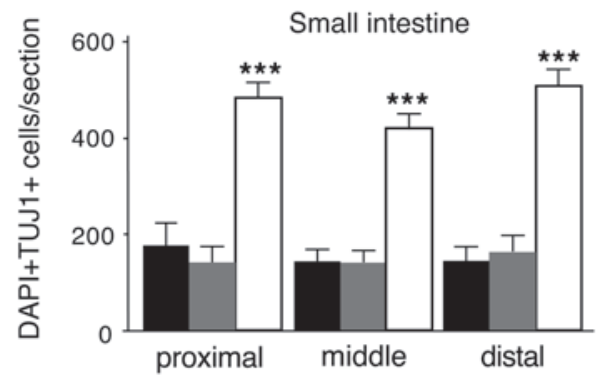

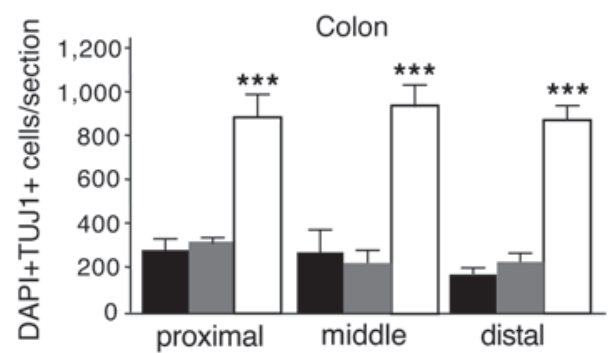

Figure 3

Hyperplasia and hypertrophy of HM ENCs. (A) Transverse sections of WT and HM colons at p15: H\&E staining, immunostaining with antibodies directed against TUJ1, NF, and Syn. Note that HM ganglia were bigger than WT ganglia. Scale bar: $50 \mu \mathrm{m}$. (B) Transverse sections of WT, HT, and $\mathrm{HM}$ colons at p15. The surface of ENS cells was estimated using ImageJ software (http://rsbweb.nih.gov/ij/). Note that HM ENS cells are twice as big as WT and HT ENS cells. ${ }^{* \star} P<0.001$. Scale bar: $25 \mu \mathrm{m}$. (C) Transverse sections of WT, HT, and HM small intestine and colon in the proximal, middle, and distal parts immunostained with TUJ1 antibody and counterstained with DAPI. The number of DAPI+ and TUJ1+ cells was determined in each compartment and plotted. Note that the number of DAPI+ and TUJ1+ cells is higher in the colon than in the small intestine. Scale bar: $25 \mu \mathrm{m}$. Dotted lines enclose ENS. Error bars represent mean + SEM.

expression in ENCs during embryonic development. We thus carried out double immunostaining for PTEN and TUJ1 in transverse sections of colon collected on E14.5, E15.5, and E17.5 from WT and HM embryos (Figure 6). In WT embryos, no TUJ1-positive cells were positive for PTEN on E14.5, approximately 10\% of TUJ1-posi- tive cells were positive for PTEN on E15.5, and approximately $100 \%$ of TUJ1-positive cells were also positive for PTEN by E17.5. In HM embryos, PTEN was not detected at any developmental stage, confirming the appropriate deletion of the PTEN gene in TUJ1-positive cells (Figure 6A and Supplemental Figure 8). As expected, on E15.5, 
A

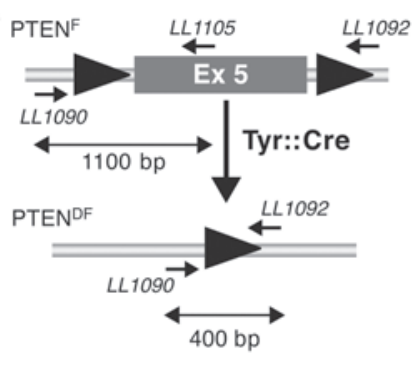

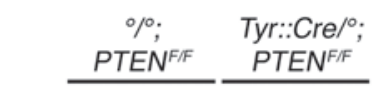

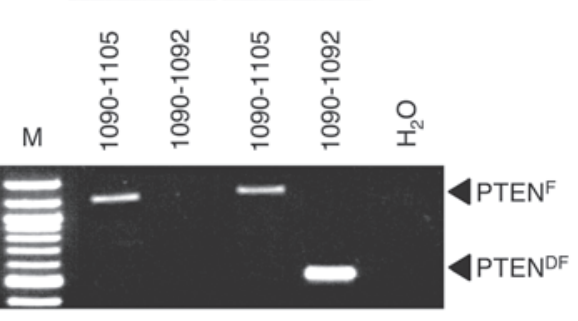

B
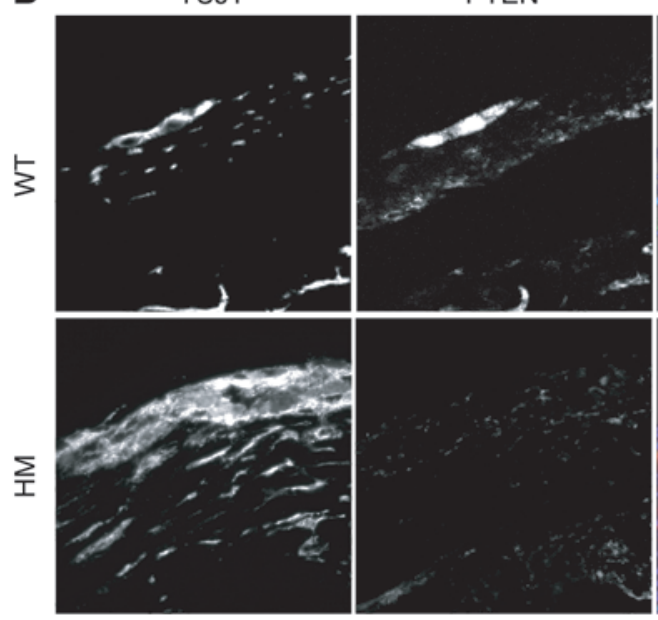

Figure 4

Conditional deletion of PTEN in ENCs. (A) Diagram, not to scale, of the floxed and defloxed PTEN locus. LoxP sequences (black triangles) flank PTEN exon 5 of the floxed allele. After appropriate crosses with Tyr:Cre transgenic mice, PTEN exon 5 is deleted in ENCs. M, marker $100 \mathrm{pb}$. (B) Transverse sections of WT and HM colons at p15, double immunostained for TUJ1 and PTEN. Note that TUJ1+ cells in the HM colon do not contain any PTEN protein. Scale bar: $25 \mu \mathrm{m}$.

epithelial cells from the mucosa were PTEN-positive and TUJ1-negative (32). We counted TUJ1-positive cells on transverse sections of colon from WT and HM embryos obtained between E14.5 and E18.5. In WT embryos, the number of TUJ1-positive cells increased between E14.5 and E16.5 and thereafter remained constant until E18.5; in HM embryos, the number of TUJ1-positive cells continued to increase throughout development, particularly between E16.5 and E18.5 (Figure 6, B and C).

The hyperplasia observed in the ENCs of HM embryos at E17.5 led us to study proliferation and the cell cycle in these cells. TUJ1 has previously been shown to be present during the final cell divisions of neuronal precursors, immediately after cell-cycle exit, and at the beginning of neuronal differentiation (39-42). We double stained transverse colon sections from E16.5 WT and HM embryos for TUJ1 and Ki67, a marker of cycling cells, or used BrdU incorporation assays to evaluate the rate of proliferation (Figure 6D). Both of these methods showed that the proportions of proliferating and cycling cells were higher in HM embryos than in WT embryos. Hypertrophy of ENCs was observed after birth but not during embryonic development (Figure 3B and Supplemental Figure 9). This suggests that hypertrophy occurs after hyperproliferation. We also examined the possibility that a reduction in ENC apoptosis contributed to the hyperplasia in the ENS in HM embryos. We immunostained transverse sections of colon from
WT and HM embryos at E15.5 and E17.5 for both activated caspase- 3 and for TUJ1 (Supplemental Figure 10). All TUJ1-positive cells were negative for activated caspase- 3 staining. ENCCs colonize the entire gastrointestinal tract between E9.5 and E13.5 (43); abnormalities in this colonization have been implicated in intestinal pseudoobstruction phenotypes $(44,45)$. Therefore, we determined whether HM embryos displayed abnormalities in ENCC migration during development by whole-mount immunostaining for TUJ1. We did not detect any differences in ENCC colonization of the gut on E12.5 and E14.5 between WT and HM embryos (Supplemental Figure 11). These results strongly suggest that the high TUJ1-positive cell count in HM embryos was due to increased proliferation during late stages of development.

We immunostained transverse sections of colon from E18.5 WT and HM embryos for both Sox10 and fatty acid-binding protein (BFABP) (Supplemental Figure 12). Sox10 is known to be produced in ENCCs and glial cells, and BFABP is produced in glial cells. The number of Sox10- and BFABP-positive cells was greater in mutants than in the WT intestine. Double immunostaining for TUJ1 (neurons) and GFAP (glial cells) was performed on p15 WT and mutant intestines. We found that the number of glial cells was greater in the p15 mutant than in p15 WT mice (Supplemental Figure 6). These results suggest that the cellular composition of the ENS was not subject to any qualitative differences (in the presence of neurons and glial cells) between mutant and WT mice. Moreover, our findings show that the absence of PTEN does not favor the existence of a particular differentiation pathway, producing either more neurons or more glial cells.

AKT inbibition during development inhibits ENS byperplasia. We found that AKT was phosphorylated in PTEN-deleted ENCs (Figure 5). To assess the role of AKT signaling in ENS development in vivo, we used an AKT inhibitor, API2. We injected pregnant females intraperitoneally with API2 on E15.5, E16.5, and E17.5. On E18.5, we counted the number of TUJ1-positive cells on transverse colon sections from WT and HM embryos from females treated and not treated with API2 (Figure 7). The numbers of TUJ1-positive cells in embryos treated with API2 did not significantly differ between WT and HM, whereas the number of TUJ1-positive cells in untreated HM embryos was double that in untreated WT embryos. Interestingly, the numbers of TUJ1-positive cells were similar between API2-treated and untreated WT embryos.

PTEN expression is abnormally low in ENS in cases of human intestinal pseudoobstruction syndrome. We assessed whether this mouse model of intestinal pseudoobstruction is relevant to the human situation. We used immunohistochemistry to analyze PTEN expression in 7 samples derived from patients presenting a diffuse GNM (neuronal hyperplasia form of CIPO) with no signs of MEN2B or NF1 and in samples obtained from 2 independent normal colons as controls. PTEN expression in the ENS was significantly lower 
A

A $\quad \mathrm{p}-\mathrm{S} 6$

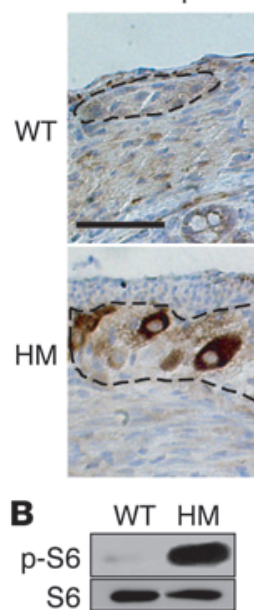

$\mathrm{S} 6$

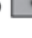

\section{- 6}

\section{p-AKT}
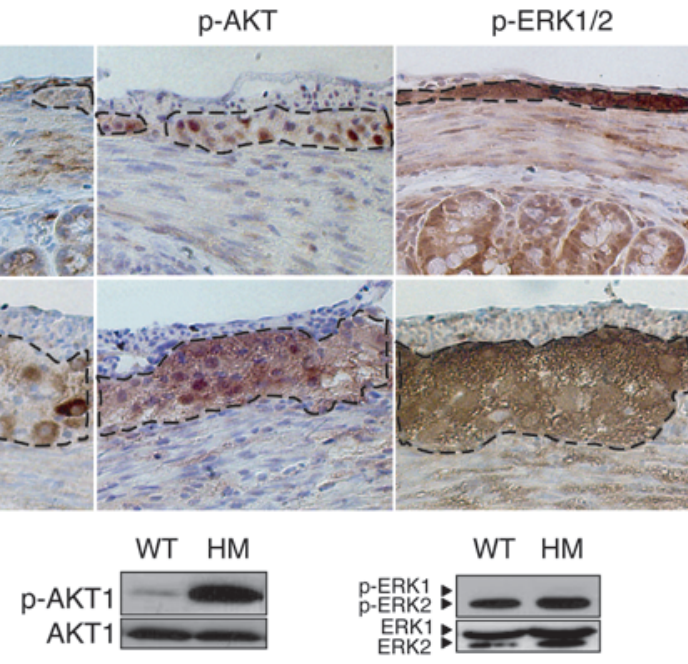

Figure 5

Deletion of the PTEN gene leads to the phosphorylation of AKT and S6. (A) Transverse sections of WT and HM p15 colon, immunostained for p-S6, p-AKT1m, and p-ERK1/2. Scale bar: $50 \mu \mathrm{m}$. Dotted lines enclose ENS. (B) Protein lysates of the WT and HM tunica muscularis from the colon were analyzed by Western blotting using antibodies directed against S6, AKT1, ERK1/2, and their phosphorylated forms. Note that ERK2 is mainly phosphorylated even though ERK1 is abundant in colon. Western blot analysis was performed for 3 WT and 5 HM colons. Representative immunohistochemical and Western blot analyses are presented.

in 2 out of 7 CIPO patients compared with normal ENS (Figure 8). Concomitantly, the level of S6 phosphorylation was substantially increased in the ENS of these 2 patients (Figure 8). As a control, we confirmed that PTEN and p-S6 immunostainings were similar in the mucosa of the 9 studied colons (data not shown). These results suggest that the PI3K/PTEN-AKT-S6K signaling pathway may be induced in patients with a neuronal hyperplasia form of CIPO with no signs of MEN2B or NF1.

\section{Discussion}

CIPO is a neurocristopathy associated with a hypo- or hyperganglionosis. In healthy individuals, the exact number of neurons and glial cells generating the ganglia and, in consequence, the ENS has to be tightly regulated. During development, the ENS is established through precise spatial and temporal molecular mechanisms that act to allow proper proliferation of these cells until they reach their optimal number. In this study, we investigated the involvement of PTEN during the development of the myenteric plexus of the gut. We evaluated the presence of PTEN in the ENS and studied the PTEN signaling pathways associated with the proliferation and growth of neuronal cells forming the ENS. For this work, we produced an animal model recapitulating human diffuse GNM.

PTEN controls the number and size of ENCs. PTEN is produced in the human ENS during the 17th week of gestation, 8 to 9 weeks after complete gut colonization by neural crest cell derivatives (33). PTEN is ubiquitously expressed in embryos during early development (E7-E11). To confirm these previous observations, we analyzed cryosections from E10.25 Tyr:Cre $/{ }^{\circ}$;Rosa26R/+ embryos by X-gal and immunostaining for PTEN. We showed that PTEN was ubiquitously produced in floxed and defloxed cells. This strongly suggests that PTEN may be defloxed in ENS cells from E9.5 (Supplemental
Figure 13). PTEN was not detected in TUJ1-positive cells (i.e., ENCs) in E14.5 embryos but was present in these cells from E15.5 (Figure 6A). Altogether, it appears that PTEN is produced in precursor cells of ENS (around E9.5-E10.5), then is repressed until E14.5; PTEN finally reappears from E15.5. Thus, PTEN starts to be produced after gastrointestinal tract colonization by neural crest cell derivatives, and it remains present in these cells after birth (Figure 1). The relative temporal pattern of PTEN expression in this lineage is conserved between mice and humans.

PTEN is first produced on E15.5 in ENCs (Figure 6), and migration of ENCs ends by E13.5 (43), suggesting that the absence of PTEN does not affect the migration of these cells. We confirmed this and demonstrated that the number and size of ENS cells are greatly affected by the absence of PTEN (Figure 3 and Supplemental Figure 11).

PTEN regulates cell number by inhibiting cell-cycle progression and inducing apoptosis (23). After E15.5, PTEN-deleted ENCs proliferated more actively than WT ENCs (as assessed by Ki67 assay and BrdU pulse analysis; Figure 6). We confirmed these findings, using another neuronal marker $(\mathrm{HuC} / \mathrm{D})$ present in the nucleus and the cytoplasm of early neurons together with the nuclear marker Ki67. This double immunostaining allowed us to unambiguously identify Ki67-positive ENCs. As expected, we obtained similar results for TUJ1/Ki67 and HuC/D/Ki67 immunostainings (Supplemental Figure 14). These results are in agreement with other findings. Indeed, ES cells lacking PTEN display early entry into S phase (23); additionally, neuronal stem/progenitor cells lacking PTEN show hyperproliferation and enhanced self renewal, suggesting that PTEN may also modulate $\mathrm{G}_{0} / \mathrm{G}_{1}$ transition (30). We did not detect any staining for caspase-3 in WT or HM ENCs at any developmental stage analyzed (Supplemental Figure 10). Our findings for WT ENCs are perfectly consistent with those of a previous study (4), showing the absence of detectable caspase- 3 in ENS cells at E12-E18, P0, P7, P14, and P60. These authors concluded that, in contrast to most other components of the nervous system, programmed cell death is not important for determining enteric neuron cell numbers. In our study, we cannot exclude that apoptosis is induced by a molecular mechanism independent of caspase-3.

We also demonstrated that PTEN mice present a hyperplasia of enteric neuronal and glial cells, suggesting that the absence of PTEN in ENS precursors does not result in the production, via the differentiation pathway, of more neurons or more glial cells.

PTEN controls translational mechanisms through regulation of mTOR/S6K signaling, thus influencing cell size (30). During development, the size of PTEN-positive ENCs was not affected (Supplemental Figure 9). We observed clear hypertrophy of these cells after birth, at $\mathrm{p} 15$, when proliferation occurs at a far lower rate (Figure 3B). These results suggest that PTEN acts in a cell determination status-dependent manner in the ENS. These results are in agreement with previous studies performed with neural stem cells before and after differentiation (30).

Thus, PTEN inactivation does not seem to have marked effects on ENS cells at E9.5, similar numbers of these cells being observed in WT and mutant E14.5 and E15.5 embryos. These results strongly suggest that PTEN has a major role in ENS proliferation during fetal development (from E14.5), but not during organogenesis (from E8.5 to E14.5). 
A
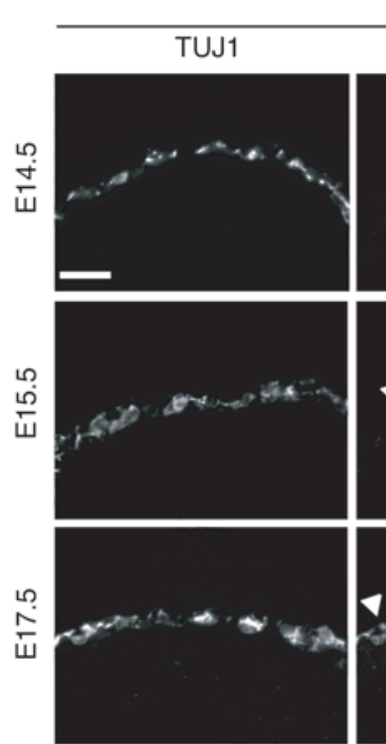

B

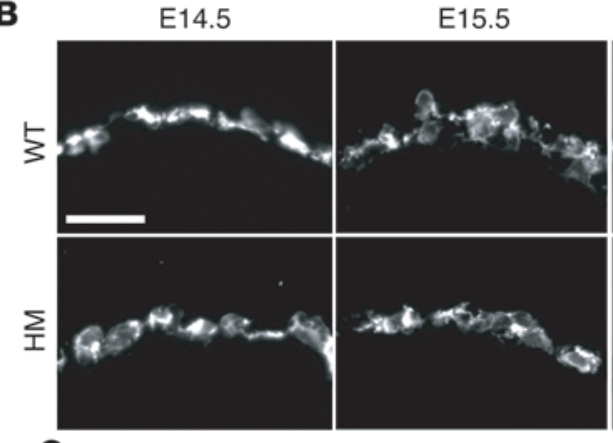

C

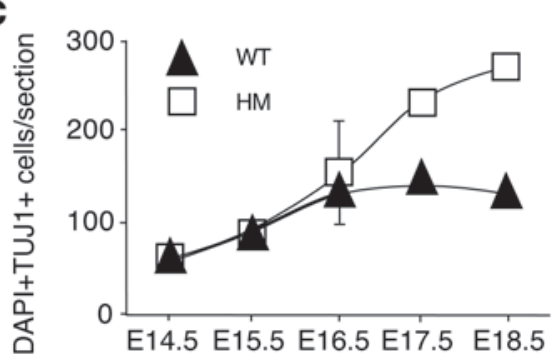

WT
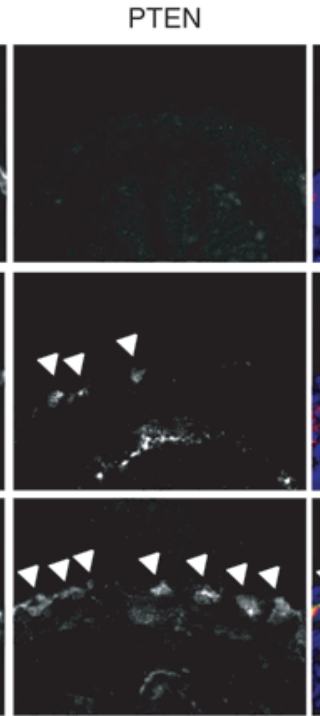

E15.5

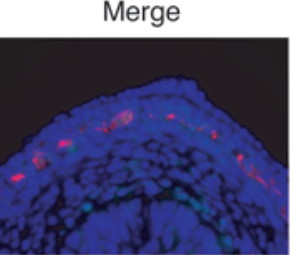

$\frac{\text { HM }}{\text { PTEN }}$
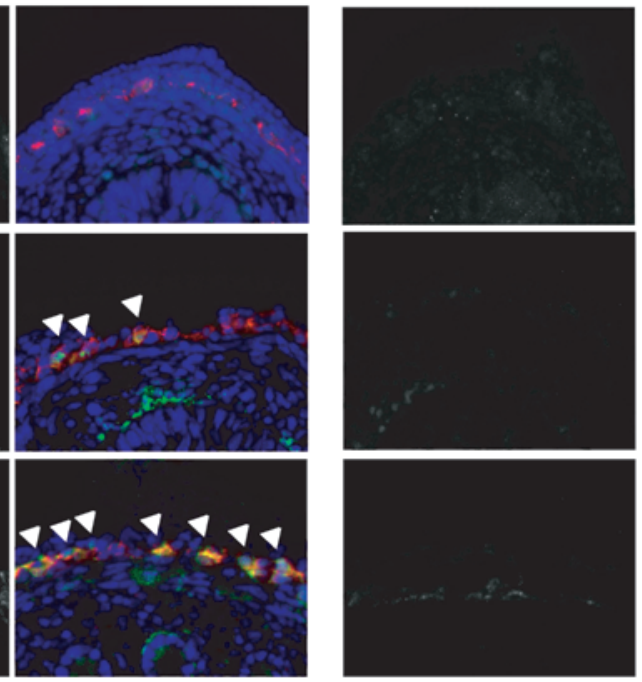

E16.5

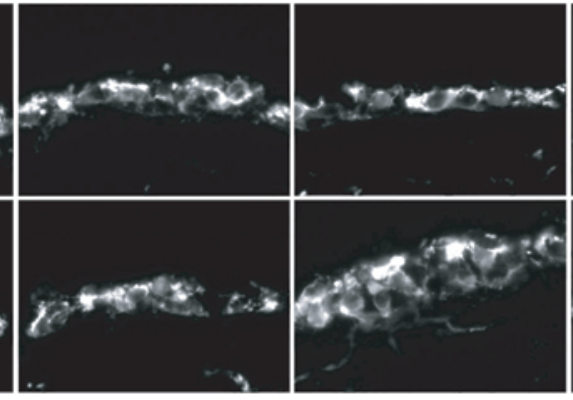

E17.5 E18.5

D

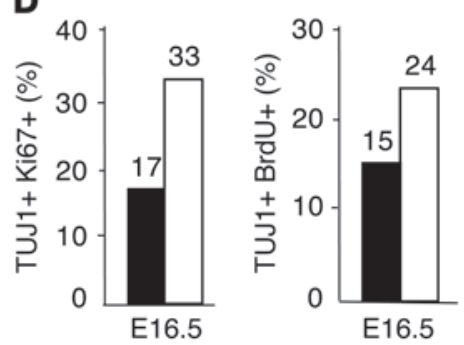

\section{Figure 6}

The rate of proliferation of ENCs carrying the PTEN gene deletion does not decline after E16.5. (A) Transverse sections of WT and HM colons at E14.5, E15.5, and E17.5 were double immunostained with antibodies directed against TUJ1 and PTEN. Merged immunofluorescence for WT colons is shown. Note that PTEN is first produced in WT ENCs at E15.5. ENCs deleted for PTEN continue to produce TUJ1 (see Supplemental Figure 4). Scale bar: $20 \mu \mathrm{m}$. Arrowheads indicate TUJ1 and PTEN double-labeled cells. (B) Transverse sections of WT and HM colons at E14.5-E18.5 were immunostained with antibody directed against TUJ1. Scale bar: $20 \mu \mathrm{m}$. (C) The number of TUJ1-positive cells is plotted against time. For each point, 3 independent embryos of each genotype were used. The SEM bar was generally smaller than the square (HM) and triangle (WT), except at E16.5. (D) Determination of the rate of cycling cells (Ki67) and proliferation rate (BrdU assay) for TUJ1-positive cells from E16.5 WT (black) and HM (white) colons. Assays were performed with 3 independent embryos of each genotype.

Importance of the PI3K/PTEN-AKT-S6K and MAPK/ERK signaling pathways in the ENS. In adult ENS mouse cells in vivo, the PI3K/ PTEN-AKT-S6K signaling pathway was weakly activated, whereas the MAPK/ERK signaling pathway displayed a high level of activity (Figure 5). Our findings in mice were in perfect agreement with observations in humans: we found that the PI3K/PTEN-AKT-S6K signaling pathway was weakly activated in human ENS cells (Figure 8 ), and previous findings have shown that the MAPK/ERK signal- ing pathway is strongly induced in ENS cells in vivo (46). PTEN can act on both the PI3K/PTEN-AKT-S6K and MAPK/ERK signaling pathways. In mice, the absence of PTEN in adult ENS cells led to the upregulation of the PI3K/PTEN-AKT-S6K signaling pathway (Figure 5). Stronger activation of this signaling pathway led to reduced amounts of p27 (Supplemental Figure 7). This observation is consistent with the previous observation of reduced p27 levels in PTEN-null ES cells (23). However, the absence of PTEN in the 
A

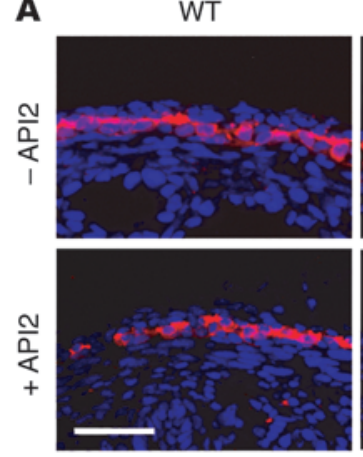

E18.5

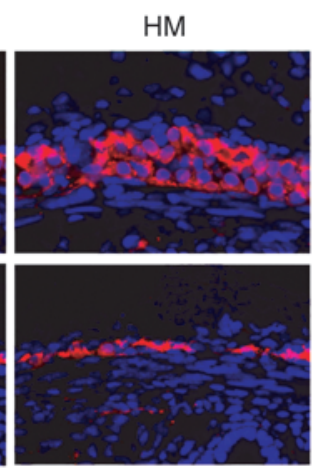

Figure 7

API2 treatment inhibits hyperplasia of HM ENCs during development. (A) After API2 treatment, E18.5 colons were isolated from WT and HM embryos. Transverse sections of colons were immunostained with antibody directed against TUJ1 and counterstained with DAPI (untreated and API2 treated). Scale bar: $25 \mu \mathrm{m}$. (B) The number of DAPI+ and TUJ1+ cells was determined and plotted. API2 was injected into 3 independent pregnant females carrying WT and HM embryos. The SEM was obtained from 4 independent embryos of each genotype.

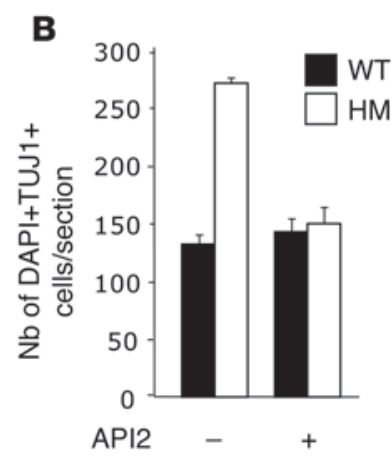

$98 \%$ of cases), in combination with NF1 (an autosomal dominant disorder, associated with NF1 mutation), or with other subtypes of GNM including Cowden syndrome (for instance, see refs. 16, 17, 49-53). Cowden syndrome is associated with a loss-of-function PTEN in $80 \%$ of the cases (31).

Thus, we initially investigated PTEN production in patients presenting with Cowden syndrome associated with GNM. Unfortunately, we were unable to obtain appropriate material for immunohistochemical analyses. However, we obtained 7 samples from patients presenting with diffuse GNM with no signs of MEN2B or NF1; 2 of these samples produced low amounts of PTEN and high levels of p-S6 (Figure 8). These results demonstrated the involvement of the PI3K/PTEN-AKT-S6K signaling pathway in diffuse GNM. We then disrupted $P T E N$ in murine ENCs in vivo to generate PTEN mice. These mice displayed some dif-

ENS did not appear to further induce the MAPK/ERK signaling pathway, which was already highly active in WT ENS. To investigate the importance of the PI3K/PTEN-AKT-S6K signaling pathway in regulating ENC proliferation in PTEN mice, we injected pregnant females carrying both WT and mutant embryos with API2 (AKT inhibitor) and determined the number of ENS cells in embryos. The numbers of ENS cells were similar in the resulting WT and mutant embryos (Figure 7). This strongly suggests that the PI3K/PTENAKT-S6K signaling pathway was the main pathway involved in the control of the proliferation of ENS cells. We tried to generate pups for API2 treatment but, unfortunately, all pups died during delivery. These results confirmed a previous in vitro analysis in which the deletion of AKT in PTEN-null ES cells completely reversed their growth advantage over WT cells, further supporting the essential role of AKT in PTEN-controlled ES cell proliferation (47).

A mouse model for buman GNM. CIPO includes at least 2 disorders: IND B and GNM (6). IND B is a human congenital disorder consisting of hyperplasia of the submucosa plexus and formation of giant ganglia, without hypertrophy of ENS cells. Two IND mouse models, HOX11L1 $1^{-/-}$and SPRY-2-/-, have been produced $(13,14)$, displaying hyperplasia of the myenteric plexus, which leads to a megacolon and intestinal pseudoobstruction. However, HOX11L1 and SPRY-2 gene mutations have not been described in cases of human IND: either mutations of HOX11L1 and SPRY-2 genes are involved but have not been discovered or unknown genes associated with HOX11L1 and SPRY-2 are mutated/dysregulated.

GNM can be classified into 3 types: polypoid ganglioneuromas, ganglioneuromatous polyposis, and diffuse GNM. Diffuse GNM is the disseminated, nodular, intramural, or transmural proliferation of neural elements involving the enteric plexuses (myenteric and submucosal plexus) along the entire gastrointestinal tract (48). The increased ENS cell density results from an abnormal number and in some cases hypertrophy of these cells (18). The 7 patients that are analyzed in this study present disseminated, nodular, and intramural proliferation of neural elements as presented for the patient CIPO no. 3 (Supplemental Figure 15). In humans, diffuse GNM may exist as an isolated manifestation, or as a component of MEN2B (associated with a constitutive active form of RET in fuse GNM characteristics, in particular, a homogeneous increase of ENC numbers along the entire gastrointestinal tract, including in the small intestine and colon (Figure 3C). The myenteric and the submucosa plexus of these mice were affected, presenting hyperplasia and hypertrophy (Figure 3 and Supplemental Figure 4). We focused mainly on the myenteric plexus, this being the main plexus implicated in propulsion of the colon through innervation of the muscular layers. The main physiopathological effect of the lack of PTEN in ENS cells suggests an intestinal pseudoobstruction similar to diffuse GNM observed in humans (Figure 2). The intestinal pseudoobstruction is certainly due to the myenteric plexus, which disrupts normal peristalsis of the gastrointestinal tract through a substantial, noncoordinated stimulation of the smooth muscle cells by the ENCs. HOX $11 \mathrm{L1}^{-{ }^{--}}$and SPRY-2-/- mice present hyperganglionosis and hyperinnervation in the ENS, causing megacolon and intestinal pseudoobstruction $(13,14)$. The motor activity of the alimentary tract is controlled by complex mechanisms regulating the balance between inhibitory and excitatory neuronal activation. A previous immunohistochemical analysis of the enteric ganglia in Hox $11 \mathrm{~L}^{-/-}$mice showed that both inhibitory (NADPH diaphorase) and excitatory (substance $\mathrm{P}$ ) neurons are hyperinnervated (12). This hyperinnervation may cause inappropriate activation of enteric neurons and may result in a functional abnormality of the colon. PTEN mice die between the second and the third week of life. However, there are some differences between the PTEN mouse model and diffuse GNM in humans. PTEN mice present hyperplasia of the glial cells (Supplemental Figures 6 and 13). However, the information available on glial cell status in diffuse GNM in humans is very limited. Moreover, in human GNM, common symptoms include constipation, diarrhea, or bleeding. In this PTEN mouse model, we observed constipation, but major diarrhea or bleeding could not be detected.

Future studies are needed to assess whether PTEN levels are reduced and P-S6 induced in appropriate samples of patients presenting Cowden syndrome with diffuse GNM. Complementary molecular genetic studies could also be easily carried out using available NF1 flox mice (54). Another important previous finding showed that some cells of the brain are defloxed in HM mice 


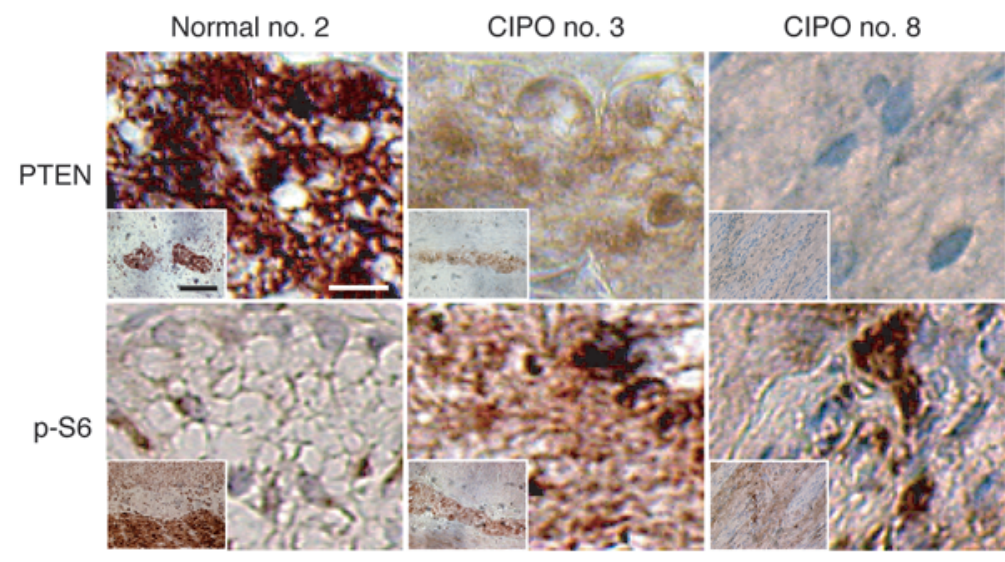

Figure 8

Levels of PTEN were substantially decreased and p-S6 was increased in human ENS presenting CIPO with no signs of MEN2B or NF1. Sections of the ENS from healthy individuals and patients presenting a neuronal hyperplasia form of CIPO were immunostained with antibodies directed against PTEN and p-S6. ENS and surrounding muscles are shown in insets. Scale bars: $10 \mu \mathrm{m} ; 100 \mu \mathrm{m}$ (inset).

(34). Consequently, some neuronal and glial cells of the brain may be deleted for PTEN, potentially affecting the hormonal balance that regulates the intestinal peristalsis. However, deletion of PTEN in granule neurons using GFAP:Cre mice does not give rise to intestinal defects (55). The somatic deletion of PTEN in these cells induces an increase of the neuronal soma size and death between 9 and 48 weeks of age. Thus, we cannot formally exclude the involvement, at least in part, of a cell nonautonomous effect in the intestinal pseudoobstruction observed in HM mice, although this seems unlikely.

In conclusion, the PTEN mouse model is the first model to allow the study of diffuse GNM, serving as a useful tool for investigating the pathogenesis of this disease. Moreover, this study implicates for the first time, to our knowledge, the PI3K/AKT/S6K signaling pathway in diffuse GNM and provides strong genetic evidence for an important role of PTEN and the PI3K/AKT/S6K signaling pathway in ENS development and homeostasis.

\section{Methods}

Mice. Floxed PTEN mice were provided by H. Wu (UCLA, Los Angeles, California, USA) and were obtained from F. Beermann (Ecole Polytechnique Fédérale de Lausanne, Lausanne, Switzerland). Characterization of the PTEN mice (30, 56), Tyr:Cre mice (34, 35, 57), Rosa26R mice (58), and ZEG mice (see Supplemental Methods) has been reported previously. Tyr:Cre transgene is located on the X chromosome (59). We did not observe any difference between mice inheriting the transgene from the mother and those inheriting the transgene paternally. No differences were observed between female mice heterozygous for Tyr:Cre/X;PTENF/F and heterozygous Tyr:Cre/Y;PTEN ${ }^{F / F}$ mice. All mice were backcrossed onto a C57BL/ 6 background for more than 6 generations. Mice were maintained in the specific pathogen-free mouse colony at the Institut Curie, in line with French and European Union law. This work obtained the approval of the Paris Ethical Committee, reference no. P2.LL.028.07. Floxed PTEN homozygous mice were crossed with HT mice to generate PTEN homozygous conditional knockouts at a frequency of $25 \%$. Mice were genotyped by PCR using DNA extracted from tails or from remaining embryonic tissue. For timed pregnancies, mice were bred and the time of plug identification was counted as day 0.5 .
Human samples. Participation of patients and their families in the study was subject to their giving informed consent and in line with the ethical guidelines of our home institution (Assistance Publique-Hopitaux de Paris; Université Paris-Descartes), with Comité de Protection des Personnes dans la Recherche Biomédicale ethical approval (decision no. 95-05-03). CIPO patients were identified either from a random survey of records or from referrals by their physicians. The phenotypic diagnosis of CIPO and the segment length involved was based on surgical, pathological, or other medical records from affected individuals presenting neuronal hyperplasia of the myenteric plexus of the colon. In 7 patients diagnosed with slow-transit constipation without HSCR, colectomy was performed and full-thickness large bowel specimens were collected (Supplemental Table 2). Full-thickness biopsy samples of age-matched control colons were obtained from pediatric patients with diseases unrelated to gastrointestinal motility disorders.

Histology and immunostaining. Mouse embryos or intestines were collected, rinsed in cold PBS, and fixed in $4 \%$ $\mathrm{PFA}$ at $4^{\circ} \mathrm{C}$ for 2 hours for embryo guts and overnight for whole embryos and intestines of adult mice. For immunohistochemistry, samples were dehydrated, embedded in paraffin wax, and sectioned into 5 - $\mu \mathrm{m}$-thick transverse sections. Sections were deparaffinized, rinsed in TBS, boiled for 20 minutes in $10 \mathrm{mM}$ sodium citrate, and treated with $3 \% \mathrm{H}_{2} \mathrm{O}_{2}$ for 30 minutes. Sections were then incubated overnight at $4{ }^{\circ} \mathrm{C}$ in TBST (TBS $/ 0.1 \%$ Tween-20) containing $5 \%$ normal goat serum and antibodies to phospho-AKT (rabbit, 1/50 dilution; Cell Signaling Technology), phospho-ERK1/2 (rabbit, 1/100 dilution; Cell Signaling Technology), phospho-S6 (rabbit, 1/75 dilution; Cell Signaling Technology), p27 (rabbit, undiluted, Abcam), TUJ1 (mouse, 1/500 dilution; Covance), NF (rabbit, 1/100 dilution; Affinity), and Syn (rabbit, 1/50 dilution; Dako). DAB (Sigma-Aldrich) was used to reveal bound antibodies according to the manufacturer's instructions. All sections were counterstained with hematoxylin. For immunofluorescence, samples were incubated for 16 hours in $30 \%$ sucrose/PBS at $4^{\circ} \mathrm{C}$, embedded in OCT compound (Sakura Finetechnical), and cut into $7-\mu \mathrm{m}$-thick sections. Sections were rinsed twice in PBT (PBS/0.1\% Tween-20), boiled for 20 minutes in $10 \mathrm{mM}$ sodium citrate, and treated with blocking solution $(2 \%$ skimmed milk in PBT) for 20 minutes at room temperature. Sections were then incubated in PBT overnight at $4^{\circ} \mathrm{C}$ with anti-TUJ1 (mouse, 1:1,000 dilution; Covance), anti-GFAP (mouse, 1:500 dilution; Cell Signaling Technology), anti-PTEN (rabbit, 1:100 dilution; Cell Signaling Technology), anti-BrdU (mouse, 1:200 dilution; BD Biosciences), and Ki67 (rabbit, 1:500 dilution; Novocastra). Alexa Fluor 488 or Alexa Fluor 555 secondary antibodies (Invitrogen) were used to detect primary antibodies. TUJ1 antibody was coupled with Alexa Fluor 488 (mouse, 1:1,000 dilution; Covance) and used for specific double immunofluorescence. See Supplemental Methods for details.

PCR analysis of recombination. PCR analysis of PTEN exon 5 was used to detect Cre-mediated recombination. Overanesthetized 2- to 3-week-old mutant and control animals were autopsied; intestines were dissected out and washed in cold PBS. The mucosae were removed from the muscularis with sharp tweezers under a dissecting binocular. DNA was extracted from a 5-mm strip of tunica muscularis of the colon and was subjected to PCR to test for floxed (primers LL1090 and LL1105) and defloxed (LL1090 and LL1092) alleles (producing fragments of 1100 and $400 \mathrm{bp}$, respectively). The sequences are as follows: LL1090, 5'-ACTCAAGGCAGGGATGAGC3'; LL1105, 5'-GTCATCTTCACTTAGCCATTGG-3'; LL1092, 5'-GCTTGATATCGAATTCCTGCAGC-3' (60). 
Western blot analysis. The muscle layer was dissected, as described above, for PCR analysis. The remaining strips of tunica muscularis were incubated in lysis buffer (50 mM Tris-HCl, pH 8; 10 mM EDTA, pH 8; 1\% SDS; $1 \mathrm{mM}$ DTT; $0.5 \mathrm{mM}$ PMSF; $5 \mu \mathrm{g} / \mathrm{ml}$ aprotinin; $2 \mu \mathrm{g} / \mathrm{ml}$ leupeptin; and $0.4 \mathrm{mM}$ sodium orthovanadate) at $95^{\circ} \mathrm{C}$ for 5 minutes and homogenized by successive passages through 18-, 20-, and 22-gauge needles. The samples were centrifuged at $20,800 \mathrm{~g}$, and the resulting cleared total cell extracts were resolved by $10 \%$ SDS-PAGE. Proteins were then analyzed by Western blot using primary antibodies to phospho-AKT (rabbit, 1:750 dilution; Cell Signaling Technology), AKT (rabbit, 1:750 dilution; Cell Signaling Technology), phospho-S6 (rabbit, 1:750 dilution; Cell Signaling Technology), S6 (mouse, 1:750 dilution; Cell Signaling Technology), phospho-ERK1/2 (mouse, 1:1000 dilution; Cell Signaling Technology), ERK1/2 (mouse, 1:1,000 dilution; Cell Signaling Technology), and TUJ1 (Supplemental Methods). Bound primary antibody was detected with horseradish peroxidase-conjugated secondary antibodies (Jackson ImmunoResearch) and SuperSignal detection (Pierce). See Supplemental Methods for details.

BrdU incorporation. BrdU (BD Biosciences) solution was injected intraperitoneally $(50 \mu \mathrm{g} / \mathrm{g}$ of animal weight for the first injection, and 20 minutes later, a second injection of $50 \mu \mathrm{g} / \mathrm{g}$ of animal weight) into pregnant mice. Embryos were harvested 2 hours after the first BrdU injection. Whole guts were dissected out, fixed for 2 hours in $4 \%$ PFA (in $1 \times$ PBS) at $4{ }^{\circ} \mathrm{C}$, and cryoprotected as described below.

API2 treatment in vivo. Pregnant female mice were injected intraperitoneally at a dose of $1 \mathrm{mg} / \mathrm{kg} / \mathrm{d}$ (dissolved in 2\% DMSO) of API2 (Calbiochem) on E15.5, E16.5, and E17.5. On E18.5, embryos were harvested and colons were dissected out, fixed for 2 hours in $4 \% \mathrm{PFA}$ at $4^{\circ} \mathrm{C}$, and cryoprotected as described above. Transverse sections and immunofluorescence were performed against TUJ1 and counterstained with DAPI as described above.

Statistics. $P$ values were determined by 2 -tailed Student's $t$ test. $P<0.05$ was considered significant.

\section{Acknowledgments}

We are grateful to H. Wu and F. Beermann (PTEN flox mice) and C. Lobe (Z/EG mice) for providing mouse strains and to $\mathrm{M}$. Wegner and T. Müller for providing Sox 10 and Bfabp antibodies, respectively. We thank J. Amiel, J. Bonaventure, S. Colombo, O. Goulet, G. Gros, and C. Rouleau for helpful discussions and materials. We thank the staff at the animal colony and the imaging facilities of Institut Curie. In particular, we are grateful to Y. Bourgeois, F. Cordelières, H. Harmange, and N. Mebirouk. I. Puig was supported by fellowships from ARC and Institut Curie. This work was supported by the Ligue Nationale Contre le Cancer (Hautde-Seine et Nationale - labellisation), INCa, cancéropole IdF. F. Jaubert and S. Lyonnet were supported by Agence Nationale de la Recherche (ANR grant MRAR HirGenet 2007) and Fondation pour la Recherche Médicale (FRM).

Received for publication May 20, 2009, and accepted in revised form September 9, 2009.

Address correspondence to: Lionel Larue, Institut Curie - Bat 110, Orsay Cedex 91405, France. Phone: 33-1-69-86-71-07; Fax: 33-169-86-71-09; E-mail: lionel.larue@curie.fr.
1. Newgreen, D., and Young, H.M. 2002. Enteric nervous system: development and developmental disturbances - part 1. Pediatr. Dev. Pathol. 5:224-247.

2. Gershon, M., Kirchgessner, A., and Wade, P. 1994. Functional anatomy of the enteric nervous system. Raven Press. New York, New York, USA. 422 pp.

3. Furness, J.B., and Costa, M. 1980. Types of nerves in the enteric nervous system. Neuroscience. 5:1-20.

4. Gianino, S., Grider, J.R., Cresswell, J., Enomoto, H., and Heuckeroth, R.O. 2003. GDNF availability determines enteric neuron number by controlling precursor proliferation. Development. 130:2187-2198.

5. Antonucci, A., et al. 2008. Chronic intestinal pseudoobstruction. World J. Gastroenterol. 14:2953-2961.

6. Kapur, R.P. 2001. Neuropathology of paediatric chronic intestinal pseudo-obstruction and related animal models. J. Pathol. 194:277-288.

7. Heanue, T.A., and Pachnis, V. 2007. Enteric nervous system development and Hirschsprung's disease: advances in genetic and stem cell studies. Nat. Rev. Neurosci. 8:466-479.

8. Burzynski, G., Shepherd, I.T., and Enomoto, H. 2009. Genetic model system studies of the development of the enteric nervous system, gut motility and Hirschsprung's disease. Neurogastroenterol. Motil. 21:113-127.

9. Borchard, F., et al. 1991. Definition and clinical significance of dysplasia in the digestive tract. Results of a meeting of the Society of Gastroenterologic Pathology of the German Society of Pathology 25 November 1989 in Kronberg [In German]. Pathologe. 12:50-56.

10. Meier-Ruge, W.A., Bruder, E., and Kapur, R.P. 2006. Intestinal neuronal dysplasia type B: one giant ganglion is not good enough. Pediatr. Dev. Pathol. 9:444-452.

11. Kapur, R.P. 2003. Neuronal dysplasia: a controversial pathological correlate of intestinal pseudoobstruction. Am J. Med. Genet. A. 122A:287-293.

12. Hatano, M., et al. 1997. A novel pathogenesis of megacolon in Ncx/Hox11L.1 deficient mice. J. Clin. Invest. 100:795-801.
13. Shirasawa, S., et al. 1997. Enx (Hox11L1)-deficient mice develop myenteric neuronal hyperplasia and megacolon. Nat. Med. 3:646-650.

14. Taketomi, T., et al. 2005. Loss of mammalian Sprouty2 leads to enteric neuronal hyperplasia and esophageal achalasia. Nat. Neurosci. 8:855-857.

15. Haggitt, R.C., and Reid, B.J. 1986. Hereditary gastrointestinal polyposis syndromes. Am. J. Surg. Pathol. 10:871-887.

16. Mulligan, L.M., and Ponder, B.A. 1995. Genetic basis of endocrine disease: multiple endocrine neoplasia type 2. J. Clin. Endocrinol. Metab. 80:1989-1995.

17. Eng, C., et al. 1996. Heterogeneous mutation of the RET proto-oncogene in subpopulations of medullary thyroid carcinoma. Cancer Res. 56:2167-2170.

18. Thway, K., and Fisher, C. 2009. Diffuse ganglioneuromatosis in small intestine associated with neurofibromatosis type 1. Ann. Diagn. Pathol. 13:50-54.

19. Wu, X., Senechal, K., Neshat, M.S., Whang, Y.E., and Sawyers, C.L. 1998. The PTEN/MMAC1 tumor suppressor phosphatase functions as a negative regulator of the phosphoinositide 3-kinase/Akt pathway. Proc. Natl. Acad. Sci. U. S. A. 95:15587-15591.

20. Li, D.M., and Sun, H. 1997. TEP1, encoded by a candidate tumor suppressor locus, is a novel protein tyrosine phosphatase regulated by transforming growth factor beta. Cancer Res. 57:2124-2129.

21. Maehama, T., and Dixon, J.E. 1998. The tumor suppressor, PTEN/MMAC1, dephosphorylates the lipid second messenger, phosphatidylinositol 3,4,5trisphosphate. J. Biol. Chem. 273:13375-13378.

22. Stambolic, V., et al. 1998. Negative regulation of $\mathrm{PKB} /$ Akt-dependent cell survival by the tumor suppressor PTEN. Cell. 95:29-39.

23. Sun, H., et al. 1999. PTEN modulates cell cycle progression and cell survival by regulating phosphatidylinositol 3,4,5,-trisphosphate and Akt/protein kinase B signaling pathway. Proc. Natl. Acad. Sci.U.S.A. 96:6199-6204.

24. Gu, J., Tamura, M., and Yamada, K.M. 1998. Tumor suppressor PTEN inhibits integrin- and growth factor-mediated mitogen-activated pro- tein (MAP) kinase signaling pathways. J. Cell Biol. 143:1375-1383.

25. Zbuk, K.M., and Eng, C. 2007. Cancer phenomics: RET and PTEN as illustrative models. Nat. Rev. Cancer. 7:35-45.

26. Salmena, L., Carracedo, A., and Pandolfi, P.P. 2008. Tenets of PTEN tumor suppression. Cell. 133:403-414.

27. Di Cristofano, A., Pesce, B., Cordon-Cardo, C., and Pandolfi, P.P. 1998. Pten is essential for embryonic development and tumour suppression. Nat. Genet. 19:348-355.

28. Podsypanina, K., et al. 1999. Mutation of Pten/ $\mathrm{Mmac} 1$ in mice causes neoplasia in multiple organ systems. Proc. Natl. Acad. Sci. U. S. A. 96:1563-1568.

29. Suzuki, A., et al. 1998. High cancer susceptibility and embryonic lethality associated with mutation of the PTEN tumor suppressor gene in mice. Curr. Biol. 8:1169-1178.

30. Groszer, M., et al. 2001. Negative regulation of neural stem/progenitor cell proliferation by the Pten tumor suppressor gene in vivo. Science. 294:2186-2189.

31. Eng, C. 2003. PTEN: one gene, many syndromes. Hum. Mutat. 22:183-198.

32. Luukko, K., Ylikorkala, A., Tiainen, M., and Makela, T.P. 1999. Expression of LKB1 and PTEN tumor suppressor genes during mouse embryonic development. Mech. Dev. 83:187-190.

33. Gimm, O., Attie-Bitach, T., Lees, J.A., Vekemans, M., and Eng, C. 2000. Expression of the PTEN tumour suppressor protein during human development. Hum. Mol. Genet. 9:1633-1639.

34. Delmas, V., Martinozzi, S., Bourgeois, Y., Holzenberger, M., and Larue, L. 2003. Cre-mediated recombination in the skin melanocyte lineage. Genesis. 36:73-80.

35. Puig, I., Yajima, I., Bonaventure, J., Delmas, V., and Larue, L. 2009. The tyrosinase promoter is active in a subset of vagal neural crest cells during early development in mice. Pigment Cell Melanoma Res. 22:331-334.

36. Asai, N., et al. 2006. Targeted mutation of serine 
697 in the Ret tyrosine kinase causes migration defect of enteric neural crest cells. Development. 133:4507-4516.

37. Carracedo, A., and Pandolfi, P.P. 2008. The PTENPI3K pathway: of feedbacks and cross-talks. Oncogene. 27:5527-5541.

38. Weng, L.P., Brown, J.L., and Eng, C. 2001. PTEN coordinates $\mathrm{G}(1)$ arrest by down-regulating cyclin D1 via its protein phosphatase activity and up-regulating p27 via its lipid phosphatase activity in a breast cancer model. Hum. Mol. Genet. 10:599-604.

39. Lee, M.K., Tuttle, J.B., Rebhun, L.I., Cleveland, D.W., and Frankfurter, A. 1990. The expression and posttranslational modification of a neuron-specific beta-tubulin isotype during chick embryogenesis. Cell. Motil. Cytoskeleton. 17:118-132.

40. Memberg, S.P., and Hall, A.K. 1995. Dividing neuron precursors express neuron-specific tubulin. J. Neurobiol. 27:26-43.

41. Moody, S.A., Quigg, M.S., and Little, C.D. 1989. Extracellular matrix components of the peripheral pathway of chick trigeminal axons. J. Comp. Neurol. 283:38-53.

42. Menezes, J.R., and Luskin, M.B. 1994. Expression of neuron-specific tubulin defines a novel population in the proliferative layers of the developing telencephalon. J. Neurosci. 14:5399-5416.

43. Young, H.M., et al. 1998. A single rostrocaudal colonization of the rodent intestine by enteric neuron precursors is revealed by the expression of Phox $2 \mathrm{~b}$, Ret, and $\mathrm{p} 75$ and by explants grown under the kidney capsule or in organ culture. Dev. Biol.
202:67-84.

44. Kapur, R.P., Yost, C., and Palmiter, R.D. 1992. A transgenic model for studying development of the enteric nervous system in normal and aganglionic mice. Development. 116:167-175.

45. Wu, J.J., Chen, J.X., Rothman, T.P., and Gershon, M.D. 1999. Inhibition of in vitro enteric neuronal development by endothelin-3: mediation by endothelin B receptors. Development. 126:1161-1173.

46. Rouleau, C., Matecki, S., Kalfa, N., Costes, V., and de Santa Barbara, P. 2009. Activation of MAP kinase (ERK1/2) in human neonatal colonic enteric nervous system. Neurogastroenterol. Motil. 21:207-214.

47. Stiles, B., et al. 2002. Essential role of AKT-1/protein kinase B alpha in PTEN-controlled tumorigenesis. Mol. Cell. Biol. 22:3842-3851.

48. Shekitka, K.M., and Sobin, L.H. 1994. Ganglioneuromas of the gastrointestinal tract. Relation to Von Recklinghausen disease and other multiple tumor syndromes. Am. J. Surg. Pathol. 18:250-257.

49. Soccorso, G., Puls, F., Richards, C., Pringle, H., and Nour, S. 2009. A ganglioneuroma of the sigmoid colon presenting as leading point of intussusception in a child: a case report. J. Pediatr. Surg. 44:e17-20.

50. Weary, P.E., Gorlin, R.J., Gentry, W.C., Jr., Comer, J.E., and Greer, K.E. 1972. Multiple hamartoma syndrome (Cowden's disease). Arch. Dermatol. 106:682-690.

51. Ortonne, J.P., Lambert, R., Daudet, J., Berthet, P., and Gianadda, E. 1980. Involvement of the digestive tract in Cowden's disease. Int. J. Dermatol. 19:570-576.
52. Lashner, B.A., Riddell, R.H., and Winans, C.S. 1986. Ganglioneuromatosis of the colon and extensive glycogenic acanthosis in Cowden's disease. Dig. Dis. Sci. 31:213-216.

53. Lacambra, C., and Gallego, M. 1998. Intestinal ganglioneuromatosis as presenting form of Cowden's syndrome in 2 brothers. Rev. Clin. Esp. 198:404-405.

54. Zhu, Y., et al. 2001. Ablation of NF1 function in neurons induces abnormal development of cerebral cortex and reactive gliosis in the brain. Genes Dev. 15:859-876.

55. Kwon, C.H., et al. 2001. Pten regulates neuronal soma size: a mouse model of Lhermitte-Duclos disease. Nat. Genet. 29:404-411.

56. Lesche, R., Groszer, M., Gao, J., Wang, Y., Messing, A., Sun, H., Liu, X., and Wu, H. 2002. Cre/loxPmediated inactivation of the murine Pten tumor suppressor gene. Genesis. 32:148-149.

57. Yajima, I., et al. 2006. Spatiotemporal gene control by the Cre-ERT2 system in melanocytes. Genesis. 44:34-43.

58. Soriano, P. 1999. Generalized lacZ expression with the ROSA26 Cre reporter strain. Nat. Genet. 21:70-71.

59. Colombo, S., Petit, V., Kumasaka, M., Delmas, V., and Larue, L. 2007. Flanking genomic region of Tyr:Cre mice, rapid genotyping for homozygous mice. Pigment Cell Res. 20:305-306.

60. Yao, D., et al. 2006. PTEN loss promotes rasHamediated papillomatogenesis via dual up-regulation of AKT activity and cell cycle deregulation but malignant conversion proceeds via PTEN-associated pathways. Cancer Res. 66:1302-1312. 